\title{
Adaptive Classification Methods for Predicting Transitions in the Nursing Workforce
}

\author{
George J. Knafl*, Mark Toles, Anna S. Beeber, Cheryl B. Jones \\ School of Nursing, University of North Carolina at Chapel Hill, Chapel Hill, NC, USA \\ Email: *gknafl@unc.edu
}

How to cite this paper: Knafl, G.J., Toles, M., Beeber, A.S. and Jones, C.B. (2018) Adaptive Classification Methods for Predicting Transitions in the Nursing Workforce. Open Journal of Statistics, 8, 497-512. https://doi.org/10.4236/ojs.2018.83032

Received: May 2, 2018

Accepted: June 10, 2018

Published: June 13, 2018

Copyright ( 2018 by authors and Scientific Research Publishing Inc. This work is licensed under the Creative Commons Attribution International License (CC BY 4.0).

http://creativecommons.org/licenses/by/4.0/

\section{(c) (i) Open Access}

\begin{abstract}
Earlier analyses of transitions from licensed practical nurse (LPN) to registered nurse (RN) in the North Carolina (NC) nursing workforce in terms of 11 categorical predictors were limited by not considering parsimonious classifications based on these predictors and by substantial amounts of missing data. To address these issues, we formulated adaptive classification methods. Secondary analyses of data collected by the NC State Board of Nursing were also conducted to demonstrate adaptive classification methods by modeling the occurrence of LPN-to-RN transitions in the NC nursing workforce from 2001-2013. These methods combine levels (values) for one or more categorical predictors into parsimonious classifications. Missing values for a predictor are treated as one level for that predictor so that the complete data can be used in the analyses; the missing level is imputed by combining it with other levels of a predictor. An adaptive nested classification generated the best model for predicting an LPN-to-RN transition based on three predictors in order of importance: year of first LPN licensure, work setting at transition, and age at first LPN licensure. These results demonstrate that adaptive classification can identify effective and parsimonious classifications for predicting dichotomous outcomes such as the occurrence of an LPN-to-RN transition.
\end{abstract}

\section{Keywords}

Adaptive Classification, LPN-to-RN Transition, LPN Workforce

\section{Introduction}

In a previous analysis of nursing workforce data modeling the occurrence of a transition from a licensed practical nurse (LPN) to a registered nurse (RN) [1], it was anticipated that study findings would inform ongoing efforts to understand the supply and behaviors of the nursing workforce. Study findings were also in- 
tended to describe potentially modifiable attributes of LPNs, who did and did not transition, that could be evaluated as opportunities for intervention. To achieve these aims, logistic regression analyses were conducted using 11 categorical characteristics as predictors, first generating unadjusted models one predictor at a time, and then generating a composite model using all 11 predictors in combination.

The data set was relatively large, with 37,781 observations. However, only two (18.2\%) of the predictors had no missing values; missing values for the other nine predictors ranged from $2(0.01 \%)$ to $7,041(18.6 \%)$. The model based on all predictors used only 27,829 (71.0\%) observations. Consequently, there was concern that missing data may have seriously affected study conclusions. Moreover, no attempt was made to remove extraneous terms from models, so generated models included non-significant terms (with $p$-values as large as 0.974 in unadjusted models and 0.958 in the composite model). To address these analysis issues, an exploratory approach was needed to systematically generate a parsimonious model using available categorical predictors while allowing for missing data and also accounting for the large sample size. Therefore, an adaptive classification approach addressing these issues was developed. This approach is presented here and demonstrated using the NC LPN workforce data.

Knafl and Ding [2] formulated and demonstrated adaptive regression methods for modeling nonlinear relationships for outcome (dependent, response) variables in terms of continuous predictor (independent, explanatory) variables. The adaptive regression modeling process is an analytic approach for conducting heuristic (i.e., rule-based) searches through power transforms of primary predictors to generate an effective model for the data. Indicator variables (i.e., dummy variables with values 0 or 1 ) as used to generate regression models equivalent to analysis of variance models can be considered in this search. For example, a categorical predictor $C$ with three levels (or values) $c_{1}, c_{2}$, and $c_{3}$ can be represented by indicator variables $I_{1}, I_{2}$, and $I_{3}$ for $C=c_{1}, C=c_{2}$, and $C=c_{3}$, respectively. The adaptive regression search can consider any subset of these three indicator variables, but does not consider automatic adjustments to those indicator variables to address combinations of their underlying sets of observations such as the indicators $I_{1,2}$ for $C=c_{1}$ or $C=c_{2}, I_{1,3}$ for $C=c_{1}$ or $C=c_{3}$, and $I_{2,3}$ for $C=c_{2}$ or $C=c_{3}$. The adaptive classification approach presented here automatically considers such combinations. A missing value is treated as one of the levels for a categorical predictor so that the complete set of observations can be used in the adaptive classification. To avoid sparse classifications with the potential for over-fitting of the data, the adaptive classification process can be restricted to consider only classifications with all of their levels occurring for at least a specific percentage of the sample size. For example, reported analyses restricted the adaptive classification search to classifications with all levels occurring for at least $5 \%$ (or 1,890) of the LPN workforce data set.

Likelihood cross-validation (LCV) scores (defined later) are used to evaluate 
and compare alternative models and to guide the adaptive classification process (as also used to guide the adaptive regression process). LCV scores for two models can be compared using LCV ratio tests, based on the $\chi^{2}$ distribution [3] and so analogous to standard likelihood ratio tests. The significance level for these tests can be controlled. For example, reported analyses adjusted for the large sample size of the NC LPN workforce data by conservatively setting the significance level $\alpha$ to 0.001 rather than to the conventional value 0.05 . The computation of LCV scores requires estimating model parameters on $k$ randomly generated subsets of the data, and so computation time increases with the sample size and with the number $k$ of subsets. For large sample sizes, computation times can be prohibitively long. However, LCV scores can be approximated for large enough sample sizes [4] by Akaike information criterion (AIC) scores [5], which can be used in such cases to reduce the computation time.

\section{LPN Data}

Data were collected annually by the NC State Board of Nursing from LPNs licensed in the state and maintained by the North Carolina Health Professions Data System at the Cecil G. Sheps Center for Health Services Research at the University of North Carolina at Chapel Hill. Data for the 2000-2013 LPN workforce were extracted from the Health Professions Data System.

Data were available for 37,781 LPNs licensed in the state of North Carolina (NC) from the years 2000 to 2013 with 3,161 (8.4\%) of these experiencing an LPN-to-RN transition between 2001-2013 as indicated by the first time presence of an $\mathrm{RN}$ license number in the data set. Data were also available for 1,617 other LPNs who had made an RN transition prior to 2001; these data were not used in reported analyses.

A total of 11 categorical characteristics were available as potential predictors of an LPN-to-RN transition (Table 1). LPNs were primarily female (93.6\%), White (69.4\%), with a degree at first LPN licensure from a US school (95.1\%), having a diploma as highest nursing degree (65.9\%), working full time (64.0\%), and residing in an urban location (73.0\%). The categories for year and age at first LPN licensure were set to approximate quartile splits for the non-missing values [1]. The nine NC Area Health Education Centers (AHECs) are listed in Table 1 in increasing size of the number of LPNs; this serves as a measure of geographical location within NC. The mission of these centers is to improve access to quality health care for the people of NC.

\section{Data Analysis}

\subsection{LCV Scores}

Models are evaluated and compared using $k$-fold likelihood cross-validation (LCV) scores. The data are first randomly partitioned into $k$ distinct subsets, called folds in the statistics literature [6]. Fold likelihoods are calculated using the data in each fold and estimates of the parameter vector $\boldsymbol{\theta}$ computed with the 
Table 1. Categorical characteristics for NC LPNs, 2000-2013.

\begin{tabular}{|c|c|c|}
\hline Characteristic & Values & $N(\%)^{\mathrm{a}}$ \\
\hline \multirow{2}{*}{ gender } & female & $35,356(93.6)$ \\
\hline & male & $2,423(6.4)$ \\
\hline & missing & $2(0.01)$ \\
\hline & White & $26,230(69.4)$ \\
\hline & Black & $9,153(24.2)$ \\
\hline \multirow{5}{*}{ race/ethnicity } & American Indian & $532(1.4)$ \\
\hline & Hispanic & $538(1.4)$ \\
\hline & Asian & $365(1.0)$ \\
\hline & other & $651(1.7)$ \\
\hline & missing & $312(0.8)$ \\
\hline \multirow[t]{6}{*}{ year of first LPN licensure } & $1938-1981$ & $8,515(22.5)$ \\
\hline & $1982-1995$ & $9,923(26.3)$ \\
\hline & $1996-2004$ & $9,017(23.9)$ \\
\hline & $2005-2013$ & $10,029(26.5)$ \\
\hline & missing & $237(0.8)$ \\
\hline & $16-22$ & $9,182(24.3)$ \\
\hline \multirow{2}{*}{ age at first LPN licensure (years) } & $23-27$ & $9,227(34.4)$ \\
\hline & $28-34$ & $9,527(25.2)$ \\
\hline \multirow{5}{*}{$\begin{array}{l}\text { degree at first LPN licensure from US } \\
\text { school }\end{array}$} & $34-68$ & $9,546(15.3)$ \\
\hline & missing & $299(0.8)$ \\
\hline & no & $1609(4.3)$ \\
\hline & yes & $35,948(95.1)$ \\
\hline & missing & $224(0.6)$ \\
\hline \multirow{4}{*}{$\begin{array}{l}\text { highest nursing degree in last year as } \\
\text { an LPN }\end{array}$} & diploma & $24,889(65.9)$ \\
\hline & associate degree & $3,688(9.8)$ \\
\hline & baccalaureate of science in nursing & 555 (1.5) \\
\hline & master of science in nursing & $281(0.7)$ \\
\hline \multirow{8}{*}{ work setting in last year as an LPN } & doctorate in nursing & $25(0.1)$ \\
\hline & missing & $8,343(22.1)$ \\
\hline & hospital in-patient & $3,748(9.9)$ \\
\hline & long-term care & $12,911(34.2)$ \\
\hline & $\begin{array}{l}\text { solo/group practice or hospital } \\
\text { out-patient }\end{array}$ & $5,711(15.1)$ \\
\hline & other & $8,458(22.4)$ \\
\hline & missing & $6,953(18.4)$ \\
\hline & community-based practice & $3,412(9.0)$ \\
\hline \multirow{3}{*}{ specialty in last year as an LPN } & geriatrics & $11,849(31.4)$ \\
\hline & medical/surgical & $1,970(5.2)$ \\
\hline & pediatrics & $2,175(5.8)$ \\
\hline \multirow{5}{*}{$\begin{array}{l}\text { employed full time in last year as an } \\
\text { LPN }\end{array}$} & other & $11,335(30.0)$ \\
\hline & missing & $7,041(18.5)$ \\
\hline & no & $6,777(1.9)$ \\
\hline & yes & $24,176(64.0)$ \\
\hline & missing & $6,828(18.1)$ \\
\hline
\end{tabular}




\section{Continued}

\begin{tabular}{ccc}
\hline located in rural area in last year as an & no & $24,579(73.0)$ \\
LPN & yes & $10,202(27.0)$ \\
& Area L & $1,398(3.7)$ \\
& South East & $2,302(6.0)$ \\
located in NC AHEC in last year as an & Greensboro & $3,497(9.3)$ \\
LPN & Mountain & $4,120(10.9)$ \\
& Southern Regional & $4,744(12.6)$ \\
& Eastern & $4,819(12.8)$ \\
& Charlotte & $5,533(14.6)$ \\
& Wake & $5,555(14.7)$ \\
& Northwest & $5,813(15.4)$ \\
\hline
\end{tabular}

AHEC-Area Health Education Center; LPN-licensed practical nurse; NC-North Carolina; US-United States. ${ }^{\mathrm{a} O u t}$ of 37,781 LPNs.

rest of the data (hence the cross-validation). Fold likelihoods are multiplied together and the product is normalized by the sample size $n$ (i.e., by raising it to the power $1 / n)$ to generate LCV scores with larger values indicating better models. The same initial seed for starting the random number generation is used to randomly generate the folds for all models for the same data so that associated LCV scores are based on the same fold assignments and so are comparable.

Larger LCV scores do not necessarily indicate substantially (or distinctly or significantly) better models. This issue of a substantial improvement in the model can be addressed with LCV ratio tests. Let $M$ denote a model for some data with $n$ observations and $M^{\prime}$ a submodel with DF fewer parameters. DF is the associated degrees of freedom for a LCV ratio test between models $M$ and $M$, and

$$
D=2 \cdot \log \left(\operatorname{LCV}(M)^{n}\right)-2 \cdot \log \left(\operatorname{LCV}(M)^{n}\right)
$$

is approximately $\chi^{2}$ distributed with DF degrees of freedom (the power $n$ is needed to remove the normalization of the LCV score by the power $1 / n$ ). As for standard likelihood ratios, the log transform is required to produce an asymptotic $\chi^{2}$ distributed statistic. This can be expressed in terms of the associated proportional decrease in the LCV score

$$
\operatorname{PD}(D, n)=\left(\operatorname{LCV}(M)-\operatorname{LCV}\left(M^{\prime}\right)\right) / \operatorname{LCV}(M)=1-\exp (-D /(2 \cdot n)) .
$$

The proportional decrease $\operatorname{PD}(D, n)$ is substantial (or distinct or significant) if it exceeds the threshold $\mathrm{PD}(\delta(1-\alpha, \mathrm{DF}), n)$ where $\delta(1-\alpha, \mathrm{DF})$ is the cutoff for a significant $\chi^{2}$ test with DF degrees of freedom and significance level $\alpha$. Equivalently, substantial improvements can be assessed using the percent decrease $\mathrm{PD}(D, n) \cdot 100 \%$ in place of the proportional decrease.

When the sample size is large, LCV scores can be approximated by AIC scores [4] formulated so that larger scores indicate better models and normalized by the sample size. Specifically, the usual smaller is better AIC score for model $M$ with estimated parameter vector $\theta$ is defined as 


$$
\operatorname{AIC}(M(\theta))=-2 \cdot \log (\mathrm{L}(\boldsymbol{\theta}))+2 \cdot \operatorname{dim}(\boldsymbol{\theta})
$$

where $\mathrm{L}(\boldsymbol{\theta})$ is the likelihood for the data evaluated at the estimated parameter vector $\boldsymbol{\theta}$ and $\operatorname{dim}(\boldsymbol{\theta})$ is the dimension of that parameter vector equaling the number of model parameters. The associated adjusted AIC score is

$$
\operatorname{AIC}^{+}(M(\theta))=\exp (-\operatorname{AIC}(M(\theta)) /(2 \cdot n)) .
$$

$\mathrm{AIC}^{+}$ratio tests can be computed similarly to LCV ratio tests. Knafl and Ding ([2], pp. 68-69) demonstrate that $\mathrm{AIC}^{+}$ratio tests are more conservative than standard likelihood ratio tests.

\subsection{Adaptive Adjustment of an Individual Categorical Predictor $C$}

Suppose that $C$ is a categorical predictor with $m$ nonmissing levels $c_{i}$ having indexes $i$ for $1 \leq i \leq m$. Starting with the full classification based on all $m$ levels in their own groups and missing values if any in a separate group, systematically merge pairs of levels one at a time as follows. Compute LCV scores for each possible merger of two levels for the current classification. If the nonmissing levels of $C$ are ordered (e.g., year at first LPN licensure levels), only consider mergers of consecutive nonmissing levels, for example, mergers of $c_{i}$ with $c_{i+1}$ for $1 \leq$ $i \leq m-1$ at the first stage of the process. If the nonmissing levels of $C$ are nominal (e.g., race), consider all pairs of two distinct levels for the current classification. If $C$ has missing values, also consider all mergers of the missing level with each of the nonmissing levels. If the best LCV score for pairwise mergers of the current classification's levels generates a substantial percent decrease compared to the LCV score for the current classification, stop the search and use the current classification. The associated threshold for this LCV ratio test is based on $\mathrm{DF}=1$ because the number of levels has changed by 1 . Otherwise (i.e., when the percent decrease is not substantial) continue the adjustment process using the pairwise merger generating the best LCV score. Note that when $C$ has missing values and the missing value level is merged with some other subset of nonmissing levels for $C$, the missing values have effectively been imputed as being one of the nonmissing levels in that subset.

\subsection{Adaptive Additive Adjustment of a Classification Using a Second Categorical Predictor $C^{\prime}$}

Suppose that a classification based on a categorical predictor $C$ has been adaptively generated using the above individual predictor adjustment process and that there are $m^{*}$ levels corresponding to groupings of the $m$ levels of $C$. Suppose that $C^{\prime}$ has $m^{\prime}$ nonmissing levels $c_{i}^{\prime}$ for $1 \leq i \leq m$ '. Apply the above single categorical predictor adjustment process to the levels of $C^{\prime}$ to systematically merge them while also including the $m^{*}$ levels of $C$ in the model. These additive classification models are based on an intercept, a fixed set of indicator variables for $m^{*}$ - 1 levels of the classification based on $C$ and indicator variables for 1 less than the number of levels for the current classification based on $C^{\prime}$.

Let $m^{*}$ denote the number of levels for the current classification based on $C^{\prime}$. 
The additive model decomposes each of the $m^{*}$ levels of the classification based on $C$ into $m^{*}$ levels for a total of $m^{*} \cdot m^{*}$ cells corresponding to combinations of levels for the classifications based on $C$ and $C^{\prime}$. Consequently, additive adjustments can generate composite classifications with relatively large numbers of cells. Moreover, some of these cells can be sparse containing relatively small numbers of observations. Nested adjustments (as defined next) of the levels of $C^{\prime}$, that is, applied separately within each of the $m^{*}$ levels of the classification based on $C$, can resolve these shortcomings.

\subsection{Adaptive Nested Adjustment of a Classification Using a Second Categorical Predictor $C^{\prime}$}

Suppose that a classification based on a categorical predictor $C$ has been adaptively generated using the above individual predictor adjustment process and that there are $m^{*}$ levels corresponding to groupings of the $m$ levels of $C$. Suppose that $C^{\prime}$ has $m^{\prime}$ nonmissing levels $c_{i}^{\prime}$ for $1 \leq i \leq m$ '. Apply the above single categorical predictor adjustment process to the complete set of levels of $C^{\prime}$ nested within each of the $m^{*}$ levels of the classification based on $C$. Compute LCV scores for each adjustment of a pair of levels of the current nested classification. If the best LCV score over all such nested adjustments generates a substantial percent decrease (using a LCV ratio test) compared to the LCV score for the current nested classification, stop the search and use the current nested classification. Otherwise continue the adjustment process considering further pairwise nested adjustments to the levels of the nested adjustment at the current stage of the process generating the best LCV score.

As an example, suppose the adaptive classification based on $C$ has three levels $c_{1}, c_{2}$, and $c_{3}$ and $C^{\prime}$ has four nonmissing ordinal levels $c_{1}^{\prime}, c_{2}^{\prime}, c_{3}^{\prime}$, and $c_{4}^{\prime}$ and no missing values. The first stage of the nested classification considers the 3 pairwise ordered mergers of $c_{1}^{\prime}$ with $c_{2}^{\prime}, c_{2}^{\prime}$ with $c_{3}^{\prime}$, and $c_{3}^{\prime}$ with $c_{4}^{\prime}$ nested within each of the 3 levels $c_{1}, c_{2}$, and $c_{3}$ for a total of 9 pairwise mergers. The next nested classification is the one based on the pairwise merger of these 9 with the best LCV score, assuming that score is not substantially smaller (using a LCV ratio test) than the score for the classification based on only the levels $c_{1}, c_{2}$, and $c_{3}$. Suppose that this corresponds to the merger $c_{1,2}^{\prime}$ of $c_{1}^{\prime}$ with $c_{2}^{\prime}$ nested within the level $c_{1}$. The next step in the process considers the same 6 pairwise mergers nested within the levels $c_{2}$ and $c_{3}$ as well as the 2 pairwise mergers of $c_{1,2}^{\prime}$ with $c_{3}^{\prime}$ and $c_{3}^{\prime}$ with $c_{4}^{\prime}$ nested within the level $c_{1}$. This nested adjustment process continues until the best LCV score for the next set of pairwise mergers generates a substantial percent decrease over the score for the current nested classification. If $C^{\prime}$ is nominal, than there are initially 6 possible mergers of distinct pairs of levels of $C^{\prime}$ within each of the 3 levels of the classification based on $C$ for a total of 18 pairwise mergers. When $C^{\prime}$ also has missing values, extra mergers are considered pairing the missing level of $C^{\prime}$ with each of the 4 nonmissing levels of $C^{\prime}$ within each of the 3 levels of the classification based on $C$ for a total of 12 extra pairwise mergers. 


\subsection{Handling More Than Two Categorical Predictors}

The additive and nested adjustments defined above for adaptively combining a second categorical predictor with a classification previously adaptively generated from a first categorical predictor generalizes readily to adaptively combining one more categorical predictor with a classification adaptively generated from two or more other categorical predictors.

\subsection{Adjusting an Adaptive Nested Classification}

The adaptive nested classification process only considers nested adjustments within each combination of levels of a prior classification and not across those combinations of levels. If all categorical predictors are ordinal or all nominal, the final nested classification can be adjusted by recoding it as a single classification and applying the adaptive classification process to that recoded classification. If the LCV score increases, the classification has been improved; if it decreases but not substantially, the adjusted classification is a competitive, parsimonious alternative. An example is provided in Section 4.5.

\subsection{Restricting the Search to Avoid Sparse Classifications}

With a sparse classification defined as one with at least one level containing less than a fixed percentage of the $n$ observations, continue any of the above adaptive classification searches if the current classification is sparse, even if that generates a substantial percent decrease in the LCV score. Once the current classification becomes nonsparse, it will remain that way throughout the rest of the process because levels increase in size with mergers. Apply the stopping rule for the search starting with the first nonsparse classification considered in the search.

\subsection{Computation}

All reported computations were conducted using SAS ${ }^{\circledR}$ version 9.4 (SAS Institute, Inc., Cary, NC). The adaptive classification process was implemented in a SAS macro. This macro and the SAS code used to generate the analyses are available at http://www.unc.edu/ gknafl/AdaptClass.html (accessed May 1, 2018).

\section{Results}

Reported adaptive classifications used the categorical characteristics of Table 1 to predict the occurrence of an LPN-to-RN transition. The significance level $\alpha$ was set at 0.001 to reflect the large sample size. Missing values were treated as an extra level so that all analyses used the complete data. Classifications were restricted to those with at least 5\% $(1,890)$ LPNs within each of their levels to avoid sparse classifications. The threshold for a substantial percent decrease in the LCV and $\mathrm{AIC}^{+}$scores for 37,781 observations, $\mathrm{DF}=1$, and significance level $\alpha=$ 0.001 was $\operatorname{PD}(D, n)=0.014 \%$ with $D=\delta(0.999,1)=10.82757$ (this threshold value was generated in the output of the SAS adaptive classification macro). 
Gender, race/ethnicity, work setting, specialty, and NC AHEC were nominal predictors; the other predictors were ordinal.

\subsection{Comparison of LCV and AIC+Scores}

The adaptive classification of year of first LPN licensure was used to assess computation times and the approximation of LCV scores by the $\mathrm{AIC}^{+}$score. LCV scores for $k=5,10$, and 15 folds were considered. For all three values of $k$ and also for the $\mathrm{AIC}^{+}$case, the single predictor adaptive classification process first merged the missing level with the 2005-2013 level and then stopped, generating the same 4-level classification. LCV scores rounded to five decimal digits were $0.76711,0.76709$, and 0.76708 for $k=5,10$, and 15 folds, respectively, compared to the $\mathrm{AIC}^{+}$score of 0.76708 . Clock times required for these computations increased from 23.6 minutes to 52.7 minutes, and then to 81.6 minutes for $k=5,10$, and 15 folds, respectively, compared to only 0.2 minutes for the $\mathrm{AIC}^{+}$ score.

Consequently, the adaptive classification of year of first LPN licensure was robust to the choice of score used to control the process. Also, the sample size was large enough to warrant use of the $\mathrm{AIC}^{+}$score in place of LCV scores, which reduced the computation times to an acceptable level not possible with LCV scores. Consequently, only $\mathrm{AIC}^{+}$scores were used in subsequent analyses.

\subsection{Adaptive Classification of Individual Characteristics}

Table 2 contains results for adaptive classification of the individual categorical characteristics of Table 1 for predicting an LPN-to-RN transition. Gender, race/ethnicity, and degree from a US school were not included in Table 2 because they generated constant classifications. Consequently, these three characteristics were not considered in subsequent analyses.

Odds ratios (ORs) for an LPN-to-RN transition are provided in Table 2. The reference categories were chosen so that all reported ORs are larger than 1, thereby indicating an increased chance of an LPN-to-RN transition. Confidence intervals and $p$-values were not reported for these ORs. Generated levels provided substantially different predictions of an LPN-to-RN transition due the adaptive classification heuristics; significance is thus a consequence of the analysis method and so seems inappropriate to report.

Missing values were not imputed for three characteristics: work setting, specialty, and employed full time. Imputation for the other six characteristics with missing values was primarily a result of restricting to nonsparse classifications with at least $5 \%$ of the LPNs in each level; of these six characteristics, only highest degree had more than $5 \%$ missing values (Table 1).

The adaptive classification based on the year of first LPN licensure generated the best (largest) $\mathrm{AIC}^{+}$score, and so provided the best individual prediction of an LPN-to-RN transition. Consequently, this classification was used as the initial classification for multiple characteristics assessments, both additive and nested, 
Table 2. Results of adaptive classification of individual categorical predictors of an LPN-to-RN transition from 2001-2013.

\begin{tabular}{|c|c|c|c|}
\hline Characteristic & Values & $\mathrm{OR}^{\mathrm{a}}$ & $\mathrm{AIC}^{+}$Score \\
\hline \multirow{5}{*}{ year of first LPN licensure } & $1938-1981$ & - & \multirow{4}{*}{0.76708} \\
\hline & $1982-1995$ & 5.44 & \\
\hline & $1996-2004$ & 18.1 & \\
\hline & 2005-2013 or missing & 8.55 & \\
\hline & $16-22$ & 1.21 & \multirow{4}{*}{0.75111} \\
\hline \multirow{3}{*}{ age at first LPN licensure (years) } & 23-27 or missing & 1.75 & \\
\hline & $28-34$ & 1.39 & \\
\hline & $35-68$ & - & \\
\hline \multirow[b]{2}{*}{$\begin{array}{l}\text { highest nursing degree in last year as } \\
\text { an LPN }\end{array}$} & diploma or associate degree & - & \multirow[b]{2}{*}{0.75106} \\
\hline & $\begin{array}{c}\text { baccalaureate, master or } \\
\text { doctorate in nursing or missing }\end{array}$ & 1.54 & \\
\hline \multirow{4}{*}{ work setting in last year as an LPN } & hospital in-patient & 3.98 & \multirow{4}{*}{0.75651} \\
\hline & long-term care & 1.49 & \\
\hline & $\begin{array}{l}\text { solo/group practice or hospital } \\
\text { out-patient or other }\end{array}$ & - & \\
\hline & missing & 2.47 & \\
\hline \multirow{4}{*}{ specialty in last year as an LPN } & community-based practice & - & \multirow{4}{*}{0.75413} \\
\hline & geriatrics, pediatrics, or other & 1.44 & \\
\hline & medical/surgical & 4.61 & \\
\hline & missing & 2.36 & \\
\hline \multirow{3}{*}{$\begin{array}{c}\text { employed full time in last year as an } \\
\text { LPN }\end{array}$} & yes & - & \multirow{3}{*}{0.75120} \\
\hline & no & 1.31 & \\
\hline & missing & 1.67 & \\
\hline \multirow{2}{*}{$\begin{array}{l}\text { located in rural area in last year as an } \\
\text { LPN }\end{array}$} & no & - & \multirow{2}{*}{0.75035} \\
\hline & yes & 1.29 & \\
\hline
\end{tabular}

AHEC-Area Health Education Center; $\mathrm{AIC}^{+}$-adjusted Akaike Information Criterion; LPN-licensed practical nurse; NC-North Carolina; OR—odds ratio; RN—registered nurse. ${ }^{\text {aOR }} \mathrm{OR}$ for a LPN-to-RN transition relative to the category with OR setting "-".

of an LPN-to-RN transition. Only the other seven characteristics that generated nonconstant individual adaptive classifications were considered, and these adaptively reduced classifications were used in adaptive assessments rather than the original characteristics.

\subsection{Adaptive Additive Classification of Multiple Characteristics}

Additive adjustments based on five of the seven other characteristics generated the unadjusted classification based on year of first LPN licensure; most likely due to restricting to nonsparse classifications. The two exceptions corresponded to employed full time and located in a rural area. Employed full-time was adjusted 
to the classification based on the employed full time level separate from the combined missing and employed part time levels. Located in a rural area was left unchanged.

The additive adjustment based on employed full time generated the larger $\mathrm{AIC}^{+}$score of 0.76818 , which improved on the adaptive classification based on only year of first LPN licensure with $\mathrm{AIC}^{+}$score 0.76708 (Table 2). Further adaptive additive adjustment of this 2-characteristic additive classification using located in a rural area left the 2-characteristic additive classification unadjusted; thereby selecting that 2-characteristic classification as the final choice for additive adjustments.

Under this selected 2-characteristic classification, compared to year of first LPN license in 1938-1981, the OR for an increased chance of an LPN-to-RN transition was 18.4, 8.25, and 5.73 for the cases 1996-2004, 2005-2013 or missing, and 1982-1995, respectively. Also, compared to being employed full time, the OR for an increased chance of an LPN-to-RN transition was 1.51 for being employed part time or missing.

\subsection{Adaptive Nested Classification of Multiple Characteristics}

Table 3 presents results for the 2-characterisitic adaptive nested classifications. Results for located in NC AHEC were not included because it generated the unadjusted classification based on year of first LPN licensure by itself. The other six cases generated classifications nested within year of first LPN licensure. Age at first LPN licensure was nested in two or three levels within each level of year of first LPN licensure. The other five classifications only affected one or two of the year of first LPN licensure levels, leaving the other levels for year of first LPN licensure unadjusted.

Work setting nested within year of first LPN licensure generated the best $\mathrm{AIC}^{+}$ score of 0.77028 . This improved on the adaptive classification based on only year of first LPN licensure with $\mathrm{AIC}^{+}$score 0.76708 and on the best adaptive additive classification with $\mathrm{AIC}^{+}$score 0.76818 . This 2-characteristic nested classification was used to generate 3-characteristic nested classifications based on the remaining five classifications that were nested within year of first LPN licensure.

The best 3-characteristic nested classification was based on further adjustments for age at first LPN licensure. All of the other four classifications had no effect, generating the model based on only work setting nested within year of first LPN licensure. Consequently, the adaptive nested classification search stopped with the final selected model described in Table 4. This model generated the best overall $\mathrm{AIC}^{+}$score of 0.77151 . While it is based on three characteristics, there are only two levels of nesting with work setting nested within first year of LPN licensure 1996-2004 and with age at first LPN licensure nested within year of first LPN licensure 1982-1995. The other two years at first LPN licensure levels are unaffected by work setting and by age. This model has an acceptable $c$-index (or area under the receiver operating characteristics curve) of 0.72 . 
Table 3. Results of adaptive nested classification of two categorical predictors of an LPN-to-RN transition from 2001-2013 starting from the year of first LPN licensure.

\begin{tabular}{|c|c|c|c|c|}
\hline Characteristic & Values & Year of First LPN Licensure & $\mathrm{OR}^{\mathrm{a}}$ & $\mathrm{AIC}^{+}$Score \\
\hline \multirow{10}{*}{$\begin{array}{l}\text { age at first LPN } \\
\text { licensure (years) }\end{array}$} & $16-27$ or missing & & 6.50 & \multirow{9}{*}{0.76954} \\
\hline & $28-68$ & 1938-1981 & - & \\
\hline & $16-27$ or missing & & 39.8 & \\
\hline & $28-34$ & 1982-1995 & 23.4 & \\
\hline & $35-68$ & & 8.94 & \\
\hline & $16-34$ or missing & \multirow{2}{*}{$1996-2004$} & 97.0 & \\
\hline & $35-68$ & & 63.5 & \\
\hline & $16-27$ or missing & \multirow{2}{*}{ 2005-2013 or missing } & 50.3 & \\
\hline & $28-68$ & & 35.6 & \\
\hline & - & $1938-1981$ & - & \multirow{8}{*}{0.76905} \\
\hline \multirow{7}{*}{$\begin{array}{l}\text { highest nursing } \\
\text { degree in last year } \\
\text { as an LPN }\end{array}$} & - & 1982-1995 & 5.44 & \\
\hline & $\begin{array}{c}\text { diploma or associate } \\
\text { degree }\end{array}$ & \multirow{6}{*}{ 2005-2013 or missing } & 27.5 & \\
\hline & baccalaureate, master or & & & \\
\hline & $\begin{array}{l}\text { doctorate in nursing or } \\
\text { missing }\end{array}$ & & 14.1 & \\
\hline & $\begin{array}{c}\text { diploma or associate } \\
\text { degree }\end{array}$ & & 5.73 & \\
\hline & baccalaureate, master or & & & \\
\hline & $\begin{array}{l}\text { doctorate in nursing or } \\
\text { missing }\end{array}$ & & 10.4 & \\
\hline \multirow{5}{*}{$\begin{array}{l}\text { work setting in last } \\
\text { year as an LPN }\end{array}$} & - & $1938-1981$ & - & \multirow{4}{*}{0.77028} \\
\hline & - & $1982-1995$ & 5.44 & \\
\hline & $\begin{array}{l}\text { hospital in-patient or } \\
\text { missing }\end{array}$ & & 33.5 & \\
\hline & $\begin{array}{c}\text { long-term care or } \\
\text { solo/group practice or } \\
\text { hospital out-patient or } \\
\text { other }\end{array}$ & $1996-2004$ & 12.1 & \\
\hline & - & 2005-2013 or missing & 8.55 & \multirow{6}{*}{0.76903} \\
\hline \multirow{4}{*}{$\begin{array}{l}\text { specialty in last year } \\
\text { as an LPN }\end{array}$} & - & $1938-1981$ & - & \\
\hline & - & $1982-1995$ & 5.44 & \\
\hline & $\begin{array}{l}\text { community-based } \\
\text { practice or geriatrics, } \\
\text { pediatrics, or other }\end{array}$ & \multirow[t]{2}{*}{$1996-2004$} & 13.8 & \\
\hline & $\begin{array}{l}\text { medical/surgical or } \\
\text { missing }\end{array}$ & & 31.3 & \\
\hline \multirow{5}{*}{$\begin{array}{c}\text { employed full time } \\
\text { in last year as an } \\
\text { LPN }\end{array}$} & - & 2005-2013 or missing & 8.55 & \\
\hline & - & $1938-1981$ & - & \multirow{4}{*}{0.76840} \\
\hline & - & 1982-1995 & 5.44 & \\
\hline & yes & \multirow{2}{*}{$1996-2004$} & 14.0 & \\
\hline & no or missing & & 27.0 & \\
\hline
\end{tabular}




\section{Continued}

\begin{tabular}{ccccc}
\hline & - & $2005-2013$ or missing & 8.55 & \\
& - & $1938-1981$ & - & \\
$\begin{array}{c}\text { located in rural area } \\
\text { in last year as an } \\
\text { LPN }\end{array}$ & - & $1982-1995$ & 5.44 & \\
& no & $1996-2004$ & 16.4 & 0.76737 \\
& yes & & 22.9 & \\
& - & $2005-2013$ or missing & 8.55 & \\
\hline
\end{tabular}

$\mathrm{AIC}^{+}$-adjusted Akaike Information Criterion; LPN-licensed practical nurse; NC-North Carolina; $\mathrm{OR}-$ odds ratio; $\mathrm{RN}-$ registered nurse. ${ }^{\mathrm{a}} \mathrm{OR}$ for a LPN-to-RN transition relative to the category with $\mathrm{OR}$ setting “-”.

Table 4. Final selected adaptive nested classification based on three categorical predictors of an LPN-to-RN transition from 2001-2013.

\begin{tabular}{|c|c|c|c|c|c|}
\hline $\begin{array}{c}\text { Age at First LPN } \\
\text { Licensure }\end{array}$ & $\begin{array}{l}\text { Work Setting in Last } \\
\text { Year as an LPN }\end{array}$ & $\begin{array}{l}\text { Year of First LPN } \\
\text { Licensure }\end{array}$ & $N(\%)^{\mathrm{a}}$ & $\mathrm{OR}^{\mathrm{b}}$ & $\mathrm{AIC}^{+}$Score \\
\hline- & - & $1938-1981$ & $8,515(22.5)$ & - & \\
\hline $\begin{array}{l}16 \text { - } 34 \text { years or } \\
\text { missing }\end{array}$ & - & $1982-1995$ & $7,134(18.9)$ & 6.92 & \\
\hline $35-68$ years & & & $2,789(7.4)$ & 1.84 & \\
\hline- & $\begin{array}{l}\text { hospital in-patient } \\
\quad \text { or missing }\end{array}$ & & $2,893(7.7)$ & 33.5 & 0.77151 \\
\hline- & $\begin{array}{l}\text { long-term care or } \\
\text { solo/group practice } \\
\text { or hospital } \\
\text { out-patient or other }\end{array}$ & $1996-2004$ & $6,124(16.2)$ & 12.1 & \\
\hline- & - & $2005-2013$ or missing & $10,326(27.3)$ & 8.55 & \\
\hline
\end{tabular}

\subsection{Example of an Adjusted Adaptive Nested Classification}

The 2-characteristic nested classification between year of first LPN licensure and age at first LPN licensure is based on nine levels (Table 3). These two classifications are ordinal and so the nested classification can be considered to have nine ordinal levels. When this composite classification was treated as a single classification and subjected to the adaptive classification process, an eight level classification was generated, merging the seventh level based on the 1996-2004 year of first LPN licensure level and the 35 - 68 year at first LPN licensure level with the following or eighth level based on the 2005-2013 or missing year of first LPN licensure level and $16-27$ or missing age at first LPN licensure level. The LCV score decreased from 0.76954 (Table 3) to 0.76947 with insubstantial percent decrease $0.009 \%$ (i.e., less than the threshold of $0.014 \%$ ), and so this was a competitive, parsimonious alternative classification. However, this did not affect the next stage of the adaptive nested classification process so that the final selected nested classification was still the one given in Table 4 . 


\section{Discussion}

Adaptive classification methods have been formulated and demonstrated using data on NC LPN-to-RN transitions in 2001-2013. These methods can be used to address individual categorical predictors as well as multiple categorical predictors combined through either additive or nested approaches. However, the additive approach can generate large numbers of combinations of levels for the individual predictors with sparse numbers within combinations. For the reported analyses, the nested approach produced models based on more individual predictors and with fewer combinations of levels than would be generated by the additive approach using the same number of predictors.

Adaptive classification can handle missing values without data loss by treating missing values as one more level for a categorical predictor, and in this case allowed for use of data from the complete NC LPN population (100\% of the data compared to only $71 \%$ in earlier analyses). This approach is also used with multiple adaptive regression splines [7]. In reported analyses, models based on two or more characteristics had all missing levels for those characteristics combined with a nonmissing level, thereby imputing those missing values. This may not always happen, but the adaptive classification heuristics can be restricted to guarantee that all missing value levels be combined with some other level.

Under the selected best model (Table 4), compared to LPNs with first licensure in 1938-1981, the largest OR of 33.5 for an increased chance of an LPN-to-RN transition occurs for LPNs with first licensure in 1996-2004 and having hospital in-patient or missing work setting. The next largest OR of 12.1 occurs for LPNs with first licensure in 1996-2004 and having any other work setting, followed by an OR of 8.55 for LPNs with first licensure in 2005-2013 or missing, then an OR of 6.92 for LPNs with first licensure in 1982-1995 and age at first LPN licensure 16 - 34 years or missing, and finally by an OR of 1.84 for LPNs with first licensure in 1982-1995 and age at first LPN licensure 35 - 68 years.

These adaptive classification results suggest some important conclusions about the LPN workforce data. The chance of an LPN-to-RN transition can be reasonably treated as depending entirely on three characteristics: year at first LPN licensure, work setting in last year as an LPN, and age at first LPN licensure, in order of importance. While this chance also depends individually on other characteristics (Table 2), these individual dependencies are reasonable considered to be explainable by the joint dependence on the three primary characteristics. The results of earlier analyses [1] suggest the opposite conclusion that characteristics other than these three are also of importance, but that conclusion was based on data for only $71 \%$ of the LPNs and using all available characteristics without attempting to identify a parsimonious alternative model.

The odds of a transition change with cohort based on the year of first LPN licensure (Table 2) varies from 5.44 to 18.1 compared to the earliest 1938-1981 cohort with the lowest chance of a transition. However, these cohort effects be- 
come more complex after further consideration of work setting and age (Table 4). For the 1982-1995 cohort, the odds for a transition interact with the age at first LPN licensure and are stronger for younger $(\mathrm{OR}=6.92)$ than older ages $(\mathrm{OR}=1.84)$. For the 1996-2004 cohort, the odds for a transition interact with the work setting in the last year as an LPN and are stronger for the hospital in-patient setting $(\mathrm{OR}=33.5)$ than for other settings $(\mathrm{OR}=12.1)$. For nurses in the other two cohorts, the chance of a transition is unaffected by any of the other classifications. These findings support the hypothesis that the odds of LPN-to-RN transitions are more prevalent in later cohorts than the 1938-1981 cohort, but not monotonically increasing as cohorts get more recent. Moreover, the largest odds of a transition occurred for the 1996-2004 cohort when working in a hospital in-patient setting and the next largest for the other LPNs in this cohort. Future studies designed to recruit LPNs for RN training and advancement might seek out nurses in the more recent cohorts, especially those employed in hospital in-patient, long-term care, and primary care settings.

A critical policy objective for the nursing workforce literature is the imperative to develop and sustain a sufficient supply of quality RNs. An important policy implication for the reported results is that a substantially greater effort will be needed to facilitate LPN-to-RN transitions in the workforce. These professional transitions were uncommon, occurring for only $8.4 \%$ of the NC LPNs over a 13 year period. Evidence from this study suggests that differences in age of LPN licensure and work setting have, for different cohorts, influenced LPN-to-RN transitions. Future investigators and policy makers might intervene to locate nurses in these groups and stimulate their interest in pursuing opportunities for career advancement. Qualitative research is needed to identify modifiable barriers to an LPN-to-RN transition and to better understand strategies that foster them.

\section{Limitations}

Adaptive classification methods are not directly supported by standard statistical software. However, a SAS macro has been developed to support those methods whose use only requires relatively basic knowledge of the SAS system. The formulation of these methods is based on an agglomerative or bottom-up approach combining larger sets of levels into smaller sets. A divisive or top-down approach is also possible, decomposing smaller sets of levels into larger sets, but has not yet been implemented. Reported analyses only addressed the logistic regression case with a dichotomous outcome, but the methods generalize to the ordinal regression case with an ordinal outcome with more than two outcome values, the multinomial regression case with a nominal outcome with more than two values, the linear regression case with a continuous outcome, and the Poisson regression case with a count outcome. Reported analyses also only addressed the univariate outcome case, but the methods generalize to the multivariate outcome case. Other methods could have been used instead, for example, multiple 
adaptive regression splines [7] or classification and regression trees [8]. Additional, unexamined factors are likely also to contribute to the occurrence of LPN-to RN-transitions, such as cultural, financial, pedagogical, and systemic educational barriers that may impede career advancement in this population. Further work is needed to investigate the impact of these other factors.

\section{Conclusions}

Reported analyses demonstrate that adaptive classification can identify effective and parsimonious classifications for predicting dichotomous outcomes such as the occurrence of an LPN-to-RN transition. Moreover, these methods provide novel and meaningful insights that can inform policy making and workforce planning. These methods can be used more broadly, for example, other kinds of transitions not only in the workforce context but also for patient transitions or transitions of any kind.

\section{Acknowledgements}

This project was funded through HRSA Cooperative Agreement U881HP26495: Health Workforce Research Centers Program.

\section{References}

[1] Jones, C.B., Toles, M., Knafl, G.J. and Beeber, A.S. (2018) An Untapped Resource in the Nursing Workforce: Licensed Practical Nurses Who Transition to Become Registered Nurses. Nursing Outlook, 66, 46-55. https://doi.org/10.1016/j.outlook.2017.07.007

[2] Knafl, G.J. and Ding, K. (2016) Adaptive Regression for Modeling Nonlinear Relationships. Springer International Publishing, Switzerland. https://doi.org/10.1007/978-3-319-33946-7

[3] Stone, M. (1977) An Asymptotic Equivalence of Choice of Model by Cross-Validation and Akaike's Criterion. Journal of the Royal Statistical Society Series B, 39, 44-47.

[4] Claeskens, G. and Hjort, N.L. (2009) Model Selection and Model Averaging. Cambridge University Press, Cambridge.

[5] Sclove, S.L. (1987) Application of Model-Selection Criteria to Some Problems in Multivariate Analysis. Psychometrika, 52, 333-343.

https://doi.org/10.1007/BF02294360

[6] Burman, P. (1989) A Comparative Study of Ordinary Cross-Validation, v-Fold Cross-Validation and the Repeated Learning-Testing Methods. Biometrika, 76, 503-514. https://doi.org/10.1093/biomet/76.3.503

[7] Friedman, J.H. (1991) Multivariate Adaptive Regression Splines. Annals of Statistics, 19, 1-67. https://doi.org/10.1214/aos/1176347963

[8] Breiman, L., Friedman, J.H., Olshen, R.A. and Stone, C.J. (1998) Classification and Regression Trees. CRC Press, Boca Raton, FL. 